R. J. Cohen and W. T. Sullivan, III, eds.

\title{
Search for and Protection of Astronomical Sites in Developing Countries
}

\author{
François R. Querci and Monique Querci \\ Observatoire Midi-Pyrénées, 14 Av. E. Belin, 31400 Toulouse, France \\ e-mail: querci@obs-mip.fr
}

\begin{abstract}
The archives of meteorological satellites allow pre-selection of dry sites well adapted to astronomical observations (in the visible, infrared and millimetric ranges). The GSM (Grating Scale Monitor) technique then permits qualification of them as sites for future astronomical observatories. Such sites are found in new astronomical countries or in developing countries. At the same time, their protection from light pollution and/or radio interference has to be secured. In practice, once pre-selections are made, the governments of these countries ought to be alerted, for example by the IAU and/or the UN Office for Outer Space Affairs. The local site testing through GSM should be carried out in cooperation with scientists of these countries under the umbrella of the IAU. This could be an approach to help introduce astronomy and astrophysics into developing countries.
\end{abstract}

\section{Introduction}

Knowledge of short time-scale variations of stars has made some progress due to campaigns of non-stop observations with simple manual telescopes and photometers, conducted simultaneously around the world. During the last two decades, the automation of telescopes (remote-controlled or robotic telescopes) opened the way to study variability over the entire HR diagram (e.g. Henry 1999), especially at places where simultaneous variations of different characteristic times, from hours to years, are found (AGB, RGB stars, etc.). To follow these permanent or temporary variations over months or years and to understand their origin, networks of robotic telescopes nowadays seem to be the most appropriate technology.

The first discoveries of the visible counterparts of gamma-ray bursts and of many new Near Earth Objects demonstrate that networks of robotic telescopes are now and will be powerful in many scientific fields (e.g. ROBONET, GNAT, TORUS, NORT, etc., as described in Querci and Querci (2000)). Consequently, sites for networks have to be established at various longitudes in the two hemispheres. Excellents sites (Hawaii, Northern Chile, South Pole, etc.) and very good ones (Canary Islands, South Africa, India, Uzbekistan, etc.) are already at work. But are we sure that other excellent sites do not exist elsewhere, for example, in developing countries? 
Astronomy and space science could contribute to the development of developing countries, as already seen some decades ago, in the Canary Islands, Chile and so on.

\section{A Way to Select Sites in Developing Countries}

A world-wide preliminary map of mean cloudiness (at $0.55 \mu \mathrm{m}$ ) was obtained from 12-year meteorological archives and with $250 \mathrm{~km}$ square meshes (Querci and Querci 1998a,b).

- A first step should be a cross correlation analysis between a worldwide map of high mountain summits (altitude $2400-3200 \mathrm{~m}$ or more) and a map of small-mesh meteorological archives $(2$ to $5 \mathrm{~km}$ ) on cloudiness, humidity, dust storms and light pollution. It would permit the determination of 20 to 30 new meteorological excellent or very good sites adapted to optical, infrared, or millimetric observations.

- A second step should be a detailed analysis of the local atmospheric turbulence for these 20 to 30 pre-selected sites by a seeing-monitor or by a grating scale-monitor (G.S.M.) technique (Martin et al. 1994). The registration of the parameters $L_{0}$, the wavefront outer scale, $r_{0}$, the Fried parameter, $\tau$, the speckle lifetime, and the isoplanetism angle for each of these sites should permit the selection of 8 to 10 sites besides those already classified as high quality observing sites.

- A third step should be the development of cooperation and analysis of the local facilities to implement and to maintain robotic telescopes and their equipment.

The two last steps could be a way to introduce astronomy and space science into developing countries through robotic equipment, especially the analysis of variable objects supported by hydrodynamical calculations.

Preliminary works on large meshes are in progress in many countries. Moreover, analysis with small meshes are in progress for the High Atlas Mountains (Morocco) and for Lebanese border mountains (Syria)(private communication).

\section{Protection for the Selected Sites}

In many developing countries, astronomy and space science are not developed at all and the search for sites is ignored. Consequently, potential sites might be polluted and lost for science in the future.

In a few developing countries, collaboration with astronomically-developed countries is in progress. So, site prospecting and subsequent site protection are now taken into account by national scientific authorities, contributing to the scientific and technical development of the country. 


\section{Conclusion}

Prospecting for and the protection of potential future astronomical sites are very important for astronomy and space science in the next century. These sites, of which many excellent ones are in developing countries, have been suggested based on a worldwide mean annual cloudiness map.

We take the opportunity of this IAU/UN symposium to ask the questions: could such prospecting and protection be promoted

- by each developing country individually?

- by some developing countries or new astronomical countries grouped together inside regional astronomical organizations such as the Arab Union for Astronomy and Space Science (AUASS)?

- by international astronomical organizations such as the European Southern Observatory (ESO), Cerro Tololo Inter-American Observatory (CTIO), etc.?

- by the International Astronomical Union?

- by the UN Office for Outer Space Affairs?

Encouragement and help from international organizations would be certainly decisive for developing countries, for their own scientific benefit as well as that of the international community.

\section{References}

Henry, G.W. 1999, Techniques for automated high-precision photometry of Sunlike stars, PASP, 111, 845

Martin, P., Tokovinin, A., Agabi, A., Borgnino, J., Ziad, A. 1994, G.S.M.: a Grating Scale Monitor for atmospheric turbulence measurements. I. The instrument and first results of angle of arrival measurements, Astr. Astrophys. Suppl. Ser., 108, 173

Querci, F.R., Querci, M. 1998a, A method for searching potential observing sites, in Preserving the Astronomical Windows, eds. Syuzo Isobe and Tomohiro Hirayama, A.S.P. Conf. Series vol.139, p.135

Querci, F.R., Querci, M. 1998b, The network of oriental robotic telescopes (NORT), at the WWW address: http://www.saao.ac.za/ wgssa/as2/nort.html

Querci, F.R., Querci, M. 2000, Robotic telescopes and networks: new tools for education and science, in Eighth UN/ESA Workshop on basic Space Science: Scientific Exploration from Space, Mafraq, Jordan, 13-17 March 1999, Astrophysics and Space Science, 273, 257-272 